\title{
Implementing Web Services Using PHP Soap Approach
}

\author{
https://doi.org/10.3991/ijim.v14i10.14391 \\ Mohammad Ali A. Hammoudeh $\left.{ }^{(}\right)$, Ajlan S. Al-Ajlan \\ Qassim University, Buraydah, Saudi Arabia \\ maah37@qu.edu.sa
}

\begin{abstract}
Recently, the utilization of IT in the Higher Education institutions has expanded, thus e-Learning must to turn out to be completely embedded into e-Learning and showing practice rather than the traditional approaches to e-Learning. However, this development inside e-Learning frameworks isn't yet completely realized, and the most significant difficulties to this objective are holding students inside instruction and attracting them to take part in Higher Education. One of the methodologies that have been taken to address these difficulties is to utilize the Web services approach to encourage working and participation between Higher Education institutions. Taking account that XML documents are the most commonly applied and successful type of Web services. Being PHP is the best choice for web developers, especially for Web services. This paper will present the proposed approach which aims to expand the current architecture of Web services to meet the technical requirements of the eLearning framework. It will give an outline of certain strategies that help designers to create SOAP with PHP language. It will portray how to expand PHP Sakai and Moodle to help Web services by utilizing the PHP SOAP method. Besides, it will delineate that this utilization of Web services is a direct advancement of Web programming models, and it will exhibit how PHP can be utilized as a fast and easy development tool for creating them. The spotlight of this paper is to research the expansion of Web services to support PHP Sakai and Moodle by utilizing the PHP SOAP strategy.
\end{abstract}

Keywords - PHP language, SOAP strategy, Sakai system, Moodle, Web Services, PHP SOAP Technique

\section{Introduction}

Web services allow collaborating among distributed company's external business partners. It develops the standards and protocols used to create the web as it is today, and is a set of emerging and proven communication protocols consisting of XML, SOAP, UDDI, and WSDL via HTTP [1, 2]. One of the best properties of web services is that they allow the integration of a number of applications quickly and easily and at a lower cost than ever before. It is expressed as WSDL, which is an XML-based language. The service may be deployed and discovered using UDDI, while SOAP enables vendor-neutral communications between applications via HTTP $[3,4]$. 
PHP has gotten the favoured decision for creating SOC, particularly for Web services. Servers furnish applications as services with XML data that are written in PHP works so as to execute these services and register these capacities with SOAP usage; this naturally makes the PHP capacities remotely available. The result of this service proprietors can concentrate on improving and building up the services that they need to execute it on the server. In this way, the association between the customer and the server, and the analysing of XML messages, will all be computerized. Today, there is no standard API for creating Web services applications, however there are a few strategies that can assist engineers with developing Web services. Right now, the most well-known systems are PHP SOAP, ezSOAP, PEAR SOAP, and NuSphere $[1,3,5]$.

These days, PHP is one of the simpler programming languages; it is ObjectOriented (OO) and tells designers the best way to exploit these new highlights. The PHP structure is very stable and clear; it alleviates designers from all the complexities of memory management and of dealing with the current versions of $\mathrm{C}$ language. The greater part of the guidelines and structures of PHP have been taken from the C, Perl, and Java languages for the production of a smooth and simple to-utilize language with no loss of strength $[1,2]$.

The proposed approach aims for broadening the present engineering of Web services to meet the specialized necessities of e-Learning frameworks. The point of this methodology is to clarify how we present Web services to meet the e-Learning frameworks. The most significant feature of this methodology is that it permits clients at various establishments in various nations to share material and work together by associating courses together, despite the fact that they are hosted on the distinctive eLearning framework. The fundamental point of this study is to present a strategy that supports engineers to make SOAP with PHP language. It will depict how to stretch out PHP Moodle to help web benefits by utilizing the PHP SOAP method. Furthermore, it will outline that this utilization of Web services is a clear advancement of Web programming models, and it will exhibit how PHP can be utilized as a quick and simple improvement instrument for making them. It will give a review of the most usually utilized classes and strategies in e-Learning frameworks through the execution of Web benefits so as to help PHP e-Learning frameworks by utilizing PHP SOAP.

This paper is organized as follows. A literature review of Web services and its implementations is exhibited in Section 2. Section 3 features the methodology on the most proficient method to introduce Web services to PHP Sakai and Moodle frameworks. The execution of Web services e-Learning frameworks by utilizing the PHP SOAP approach is portrayed in Section 4. At long last, the conclusion of this paper and future work is in Section 5.

\section{$2 \quad$ Literature Review}

\subsection{Web services}

Web services are quickly developing as a well-known standard for sharing data and functionality between freely Web services by utilizing the PHP SOAP approach. 
Likewise, it will delineate that this utilization of Web services is a clear advancement of Web programming models, and it will show how PHP can be utilized as a quick and simple improvement device for making them [2].

As of now, most associations utilize dissimilar applications that trade and store data in various methodologies [6]. They bolster the nuts and bolts of e-business forms that are distributed over the Internet and that exist by means of standard protocols and interfaces. Web services are a usage of SOC that gives a straightforward system to interface applications paying little heed to device or technology. They have developed as a handy, financially savvy solution for joining data appropriated between basic applications over language hindrances, operating systems, and platforms that were closed [7].

Web services are a gathering of rising and set up correspondence communication protocols that comprise of Extensible Markup Language (XML), Simple Object Application Protocol (SOAP), Universal Description Discovery and Integration (UDDI) and Web Services Description Language (WSDL) over Hypertext Transfer Protocol (HTTP). They permit applications to be incorporated quicker, more effectively and more economically than any time in recent memory. They are communicated as a WSDL that is XML-based language. Services indicate an agreement between the customer and the activities that can be expected. Services distributed and found utilizing UDDI, while SOAP permits $[8,9]$.

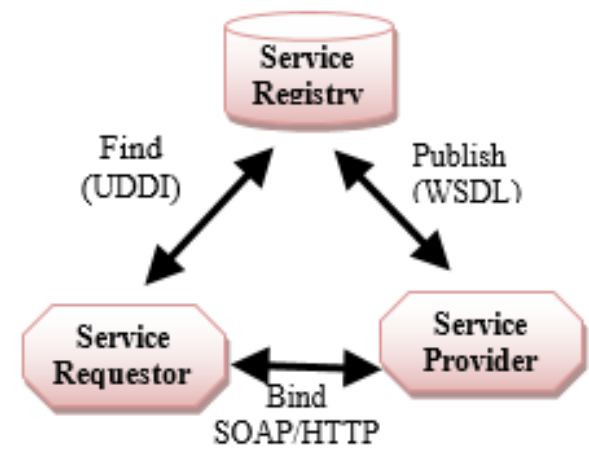

Fig. 1. The General Architectural Model of Web services

Web services architecture means to give a standard-based platform to SOC. It characterizes a lot of particulars that help an open XML-based platform for the depiction, revelation, and interoperability of disseminated, heterogeneous applications as services [10]. Web services architecture depends on the collaborations between three jobs: service provider, service registry and service requestor as in Figure 1. The connections include distribute, find and tie tasks. Together, these jobs and tasks follow up on Web services artifacts, the Web services software module and its depiction $[1,2$, $11]$. 
Service provider (SP): It is the owner of the service and it is responsible for publishing a description of its service to a service registry. It also hosts the service and controls access to it.

Service registry (specialist) (SRb): It is the main store that makes conceivable help revelation by service requestors. This segment gives an accessible storehouse of service depiction where service provider distributes their services, and where service requesters discover benefits and acquire restricting information for these services.

Service requestor (SR): It is a software part looking for the service to conjure over the Web. It finds reasonable service by finding a lot of accessible services that meet some pre-characterized criteria.

The scenario of Web service comprises of four phases, as underneath and these stages seem to be:

1. The service provider conveys and distributes a depiction of its services to the service registry, and hosts these services and controls access to them.

2. The service requester works with a service broker to find the ideal services that meet and fulfil the determinations of the register.

3. The service broker sends the services that have been found to the service requestor.

4. In the last stage, the service requester haggles with the service provider to tie the services after they have been found.

The objectives of Web services are to institutionalize the tasks, wording, ideas, message trades and the WSDL expected to communicate the warning segments and to give a language to depict points. In meeting these objectives, the Web services details should unequivocally address the utilitarian prerequisites of its clients $[14,15]$.

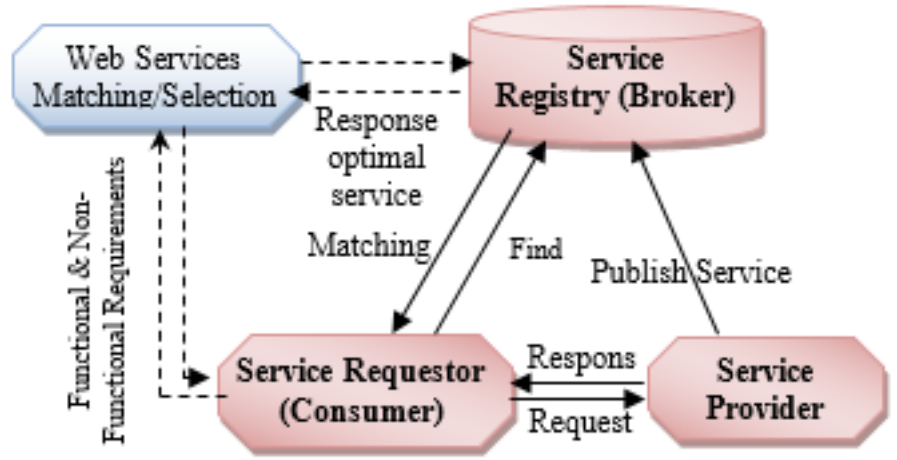

Fig. 2. The Proposed Architectural Extension Based on Web Services

In Figure 2, this investigation proposes an expansion of the current Web services system, which empowers an assortment of functional and non-functional service necessities at run-time, and utilizes the gathered data in revelation, official, and execution of a Web service. Expanding e-learning frameworks to utilize Web services has the accompanying favourable circumstances $[2,16,17]$ : 
1. It gives greater adaptability in controlling the associating activity to acquire the ideal service.

2. It lessens the determination service by utilizing the exact properties of both customer and service.

3. It is an amicable and effectively open instrument for the revelation and choice of services.

4. It assists in building a significant level of trust.

\subsection{Web service implementations}

The chart in Figure 3 delineates the scenario of two e-Learning frameworks, which are SP-1 Moodle (School-1) and SP-2 Sakai (School-2). Utilizing services in eLearning frameworks, as appeared in Figure 3, will help educators to offer their students the chance to study with more certainty and to advance in their $\mathrm{HE}$ all the more effectively and all the more rapidly. It additionally urges students to impart data to their associates in a similar organization and furthermore with different establishments over the world. The proposed framework additionally carries simple access to the educator and students outside official working hours, just as lessening the authoritative weight of the educational program through the misuse of the methods and instruments. The proposed model has a few segments, which are:

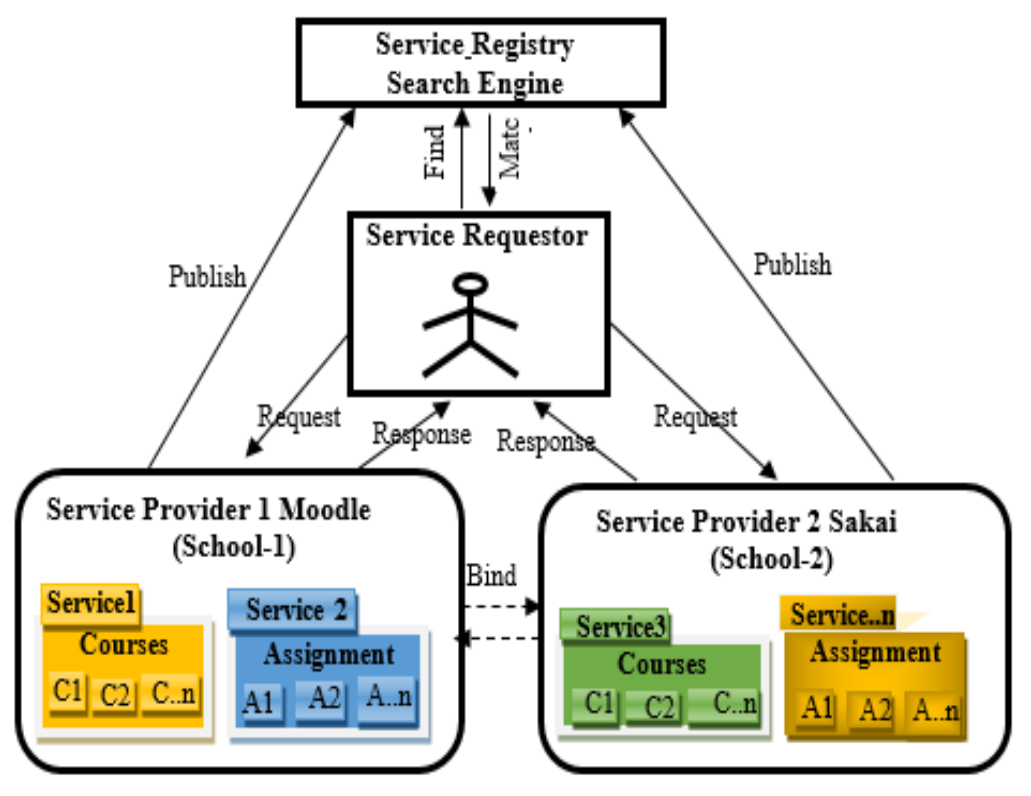

Fig. 3. Web Services in Moodle \& Sakai Systems 


\section{Results and Discussion}

This study aims mainly to demonstrate the usefulness of web services, as elearning represents services that can be published, refined, and configured using web services. It is worth noting that it is necessary to adjust the requirements of e-learning systems in order to provide a mobile and efficient environment.

PHP has become a good choice for developers using web services technology, especially in e-learning systems, so introducing web services to e-learning systems (Moodle and Sakai) is one of the main goals of this study. Recently, researchers facilitated discussion on developing web services, and as a result, we have linked that implementing web services to support PHP needs one of the techniques discussed in Section 2 of this paper. This technology is a PHP SOAP package for PHP. All functions will be registered with the PHP SOAP package, which will make the PHP functions automatically available remotely. XML messages will be generated automatically by PHP SOAP. The result will allow researchers to focus on developing the services they want to apply to the server. The communication between the client and the server was contemplated and the XML messages were analyzed mechanically.

This paper provided a total understanding of evolving WSDL and SOAP services and the clients using PHP. It indicates the most used SOAP applications applied today and ensures using the PHP SOAP package for developing SOAP applications. It's not limited to give us a good overview of how customers and servers functions communicate, but also how these XML messages are analyzed in PHP variables. Moreover, this paper summarized the classes and methods commonly used in e-learning systems by implementing web services to support PHP e-learning systems using the PHP SOAP package.

E-learning services serve five clients (supervisor, teacher, assistant teacher, student and guest) who can access their details, as stated by the user's permissions. The service owner should provide guests with information specified in the service parameters.

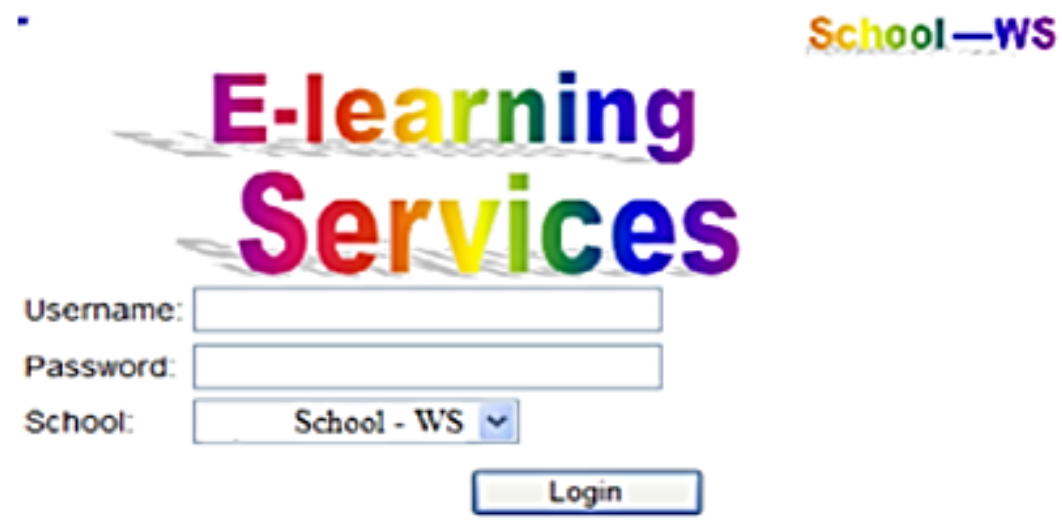

Fig. 4. Login form for the proposed model 
The user name and password for the login services must be recorded in the PHP SOAP package like all services as in Figure 4. When users access, the system stores the user details to allow them to browse all the pages that the service owner allows them to see. For extra security, deleting all user details after the user logs out is important to because data is already available in the database of their school.

As in Table 1, the system contains many files including PHP files. One of these files, Cascading Style Sheets, is a style sheet language programming used to determine the availability of a document written in a mark-up language.

Table 1. The Files in the system

\begin{tabular}{lll}
\hline No & File & Description \\
\hline 1. & server.php & $\begin{array}{l}\text { It is most important and every school has to server.php. It has a } \\
\text { complex type, registration services, and all functions with select } \\
\text { query. }\end{array}$ \\
\hline 2. & search.php & control the search in the system \\
\hline 3. & phpsoap.php & It is the main file in the system. \\
\hline 4. & school.css & Control the frame of pages and written in a mark-up language. \\
\hline 6. & course.php & It has course details. \\
\hline 7. & assignment.php & To get assignment details. \\
\hline 8. & login.php & Log in service to enable users to browse the system \\
\hline 9. & logout.php & To log out and destroy the section. \\
\hline 10. & student.php & To get student details. \\
\hline
\end{tabular}

\section{$4 \quad$ Experimental}

\subsection{The proposed approach}

Empowering Web services for various e-Learning framework applications to permit clients at multi-organizations in various nations to share material and work together by interfacing singular modules together, despite the fact that facilitated various e-Learning framework applications. In this paper, are two sorts of e-Learning frameworks applications, which both use Sakai and Moodle frameworks. Furthermore, this methodology can manage administrations with multi e-Learning frameworks applications by adding server.php document to e-Learning application and PHP SOAP to the school server $[2,21]$.

The prerequisites for the Web services e-Learning framework applications by utilizing the PHP SOAP system has the most significant segments that are fundamental in the event that one is to utilize benefits in Sakai and Moodle as in Figure.3. These significant parts will depict in more detail as follow: 
Service provider: The meaning of SP has clarified in section 2.3 above. It has the service and controls access to it. This paper has two service providers that are facilitated in better places, which are SP-1 Moodle (School - 1) and SP-2 Sakai (School 2), as in Figure.3.

SP-1 will distribute its services, all courses and related assignments in service its registry by utilizing the Moodle framework. At the point when the service requestor looks for his/her particular request, the proposed model will decrease the service choice by utilizing the properties of both client and service and offer the ideal one that addresses the requestor's issues.

SP-2 It will likewise distribute its services, all courses, and related assignments in a service registry to be accessible to a service requestor by utilizing Sakai framework. Likewise, here, the proposed model will offer the ideal one that addresses requestor's issues.

Service registry: The SRb plays a significant segment in the proposed model by utilizing the PHP SOAP bundle and fills in as a UDDI registry. This part offers an accessible archive of service depictions where service providers distribute their services, and service requesters discover benefits and acquire restricting information for these services. It draws in numerous services from suppliers and offers them, empowering requestors to discover and utilize an assortment of services that address their issues. The most significant objectives of SR are to give a just open instrument to the disclosure and distributing services, to manufacture and keep up trust in both requestor and supplier parts, and to coordinate numerous clients as conceivable to suppliers that offer services of consideration regarding the clients [1].

Service requestor: An SR is known as a service consumer, and it is a software part looking for the service to request over the Web. It finds the most reasonable service by finding the arrangement of accessible services that meet some pre-characterized criteria. A service requestor is the sender of a Web service message or the software program mentioning a particular Web service. In WSMS, the service requestor is an administrator, instructor, student, and visitor [11].

Course $\boldsymbol{\&}$ assignment details: Figure 3 above shows numerous insights concerning activities (course and task) in both School-1 (Moodle) and School-2 (Sakai), and thusly the SP in both will just distribute the essential information; they distributed the details of their activities (course and task) services, as in Table 1. Services are offered for a client as a service in the service registry (PHP SOAP bundle).

WSDL mode service: Here is the hotspot for the WSDL mode service utilizing the PHP SOAP expansion API. The PHP SOAP document ought to be put in the library's organizer named phpsoap.php. We need to unequivocally indicate which types are being utilized by the capacities, and we depict the utilitarian methodology for enrolling the sorts. The following is the WSDL arranging apportion for a PHP SOAP server. 


\section{Conclusion and Future Work}

This paper has shown the key focuses about Web services that include broadened, approximately coupled activities between e-Learning systems. Most activities can be thought of as e-Learning processes that connect with a few services in a methodology that gets about the ideal e-Learning outcome. Embracing Web services can possibly achieve diminished programming trouble and cost, lower maintenance costs, snappier time-to-showcase, new income streams, and improved operational effectiveness.

Conveying services enrolments, depictions, disclosure, and restricting has open wide the entryway for cooperative e-Learning services that run under a distributed, adaptable and effective methodology. Beside it bolsters the worldwide of offering online courses, which serve all foundations around the world with students over the internet. So as to demonstrate the idea, we have chosen a notable assistance gave by items, for example, e-Learning frameworks (Moodle and Sakai). Moreover, it has demonstrated that utilizing Web services is a basic advancement of Web programming models and has demonstrated how PHP can be utilized as a simple and quick improvement device for building them.

This paper has outlined the Web services requests to help e-Learning frameworks in fact. It has concentrated on course and task in e-Learning frameworks and how to utilize its ideas to help clients informally recognize specialized necessities and chat with others about specialized prerequisites utilizing standard wording. Finding and choosing ideal services by utilizing existing standard innovation is, for the most part, the errand of keyword-based hunts in complex service deception.

\section{$6 \quad$ References}

[1] Tyler Anderson. (2006). Build a Web service with PHP. retrieve on 10/1/2020 https://www.ibm.com/developerworks/opensource/tutorials/os-php-webservice/index.html.

[2] Dudhe, A., \& Sherekar, S. S. (2014). Performance Analysis of SOAP and RESTful Mobile Web Services in Cloud Environment. International Journal of Computer Applications, 975,8887

[3] Maleshkova, M., Philipp, P., Sure-Vetter, Y., \& Studer, R. (2019). Smart Web Services (SmartWS)--The Future of Services on the Web. arXiv preprint arXiv:1902.00910.

[4] Xinhua, E., \& Zhu, B. (2018). Big Data Service Delivery Network. In Proceedings of the 2018 International Conference on Mathematics and Statistics (pp. 89-91). https://doi.org/ $\underline{10.1145 / 3274250.3275113}$

[5] Bhuvaneswari, A., \& Karpagam, G. R. (2018). Semantic web service discovery for mobile web services.International Journal of Business Intelligence and Data Mining, 13(1-3), 95107. https://doi.org/10.1504/ijbidm.2018.088421

[6] Lee, Y. J., \& Kim, C. S. (2011). A learning ontology method for restful semantic web services. In 2011 ieee international conference on web services (pp. 251-258). IEEE. https:// doi.org/10.1109/icws.2011.59

[7] Alwasouf, A. A., \& Kumar, D. (2019). Research Challenges of Web Service Composition. In Software Engineering (pp. 681-689). Springer, Singapore. https://doi.org/10.1007/978$\underline{981-10-8848-3 \quad 66}$ 
[8] Rathod, D. (2017). Performance evaluation of restful web services and soap/wsdl web services. International Journal of Advanced Research in Computer Science, 8(7). https://doi. org/10.26483/ijarcs.v8i7.4349

[9] Nolan, D., \& Lang, D. T. (2014). Accessing SOAP Web Services. In XML and Web Technologies for Data Sciences with R (pp. 403-439). Springer, New York, NY. https://doi.org/ 10.1007/978-1-4614-7900-0_12

[10] Chen, X., Wu, T., Xie, Q., \& He, J. (2017). Data flow-oriented multi-path semantic web service composition using extended SPARQL. In 2017 IEEE International Conference on Web Services (ICWS) (pp. 882-885). IEEE. https://doi.org/10.1109/icws.2017.112

[11] Paik, H. Y., Lemos, A. L., Barukh, M. C., Benatallah, B., \& Natarajan, A. (2017). Web Service Implementation and Composition Techniques (Vol. 256). Springer International Publishing. https://doi.org/10.1007/978-3-319-55542-3

[12] Das, M. S., Govardhan, A., \& lakshmi, D. V. (2015). QoS of Web Services Architecture. In Proceedings of the The International Conference on Engineering \& MIS 2015 (pp. 1-8). https://doi.org/10.1145/2832987.2833079

[13] Boleng, J., \& Sward, R. (2013). Service-oriented architecture (SOA) concepts and implementations. ACM SIGAda Ada Letters, 33(3), 11-12. https://doi.org/10.1145/2658982. 2527289

[14] Sumathi Pawar and Niranjan N. Chiplunkar (2018). Survey on discovery of web services. Indian Journal of Science and Technology 11(16), pp. 1-10, https://doi.org/10.17485/ijst/ 2018/v11i16/120397

[15] Gottardi, T., \& Braga, R. T. V. (2017, August). Model-Oriented Web Service Implementations Compared to Traditional Web Services. In 2017 IEEE International Conference on Information Reuse and Integration (IRI) (pp. 315-324). IEEE. https://doi.org/10.1109/iri. 2017.50

[16] Das, M. S., Govardhan, A., \& Lakshmi, D. V. (2016, March). Best practices for web applications to improve performance of QoS. In Proceedings of the Second International Conference on Information and Communication Technology for Competitive Strategies (pp. 19). https://doi.org/10.1145/2905055.2905339

[17] Oliveira, R. A., Laranjeiro, N., \& Vieira, M. (2015, April). Characterizing the performance of web service frameworks under security attacks. In Proceedings of the 30th Annual ACM Symposium on Applied Computing (pp. 1711-1718). https://doi.org/10.1145/2695664.2695 $\underline{927}$

[18] Zillmer, R., Wright, R., Bates, S., \& Mahers, I. (2014). A robust device for large-scale monitoring of bar soap usage in free-living conditions. Personal and ubiquitous computing, 18(8), 2057-2064. https://doi.org/10.1007/s00779-014-0760-9

[19] Richards, Robert. (2007). Pro PHP XML and Web Services. Apress, Berkeley, CA. https:// doi.org/10.1007/978-1-4302-0139-7

[20] Rahmatulloh, A., Gunawan, R., \& Darmawan, I. (2019, March). Web Services to Overcome Interoperability in Fingerprint-based Attendance System. In 2018 International Conference on Industrial Enterprise and System Engineering (IcoIESE 2018). Atlantis Press. https://doi.org/10.2991/icoiese-18.2019.49

[21] Fadil, O. A., \& Khaldi, M. (2020). Learning Management Systems: Concept and Challenges. In Personalization and Collaboration in Adaptive E-Learning (pp. 158-175). IGI Global. https://doi.org/10.4018/978-1-7998-1492-4.ch007 


\section{Authors}

Mohammad Ali A. Hammoudeh currently works at, Department of Information Technology, College of Computer, Qassim University, Buraydah, Saudi Arabia. Email:maah37@qu.edu.sa

Ajlan S. Al-Ajlan works at Department of Management Information System \& Production Management (MISPM), College of Business and Economics, Qassim University, Buraydah, Saudi Arabia. Email: aajlan@qu.edu.sa

Article submitted 2020-03-07. Resubmitted 2020-04-11. Final acceptance 2020-04-12. Final version published as submitted by the authors. 